\title{
International Unit per Milligram of creatinine
}

National Cancer Institute

\section{Source}

National Cancer Institute. International Unit per Milligram of Creatinine. NCI Thesaurus.

Code C75903.

A unit of arbitrary substance concentration with biologic activity defined as the concentration of international unit(s) per milligram of creatinine. 